\title{
T-cell independent IgM and enduring protective IgG antibodies induced by chimeric measles viruses
}

\author{
Thomas Fehr ${ }^{1}$, Hussein Y. Naim ${ }^{2}$, Martin F. BachmanN ${ }^{1}$, Adrian F. OChSEnbein ${ }^{1}$, \\ PiUS SPIElHOFer ${ }^{2}$, ETIENNE BUCHER ${ }^{1}$, HANS HENGARTNER ${ }^{1}$, \\ Martin A. Billeter ${ }^{2} \&$ Rolf M. Zinkernagel ${ }^{1}$ \\ ${ }^{1}$ Institute of Experimental Immunology, University Hospital, Schmelzbergstrasse 12, CH-8091 Zürich Switzerland \\ ${ }^{2}$ Institute of Molecular Biology I, University of Zürich Hönggerberg, CH-8093 Zürich Switzerland \\ Correspondence should be addressed to R.M.Z.
}

\begin{abstract}
B-cell activation depends on the intensity of B-cell receptor cross-linking. Studies of haptenated antigens ${ }^{1}$ and vesicular stomatitis virus ${ }^{2}$ (VSV) have demonstrated a correlation between antigen repetitiveness and the degree to which B-cell activation is independent of $T$ cells. Here, we compare neutralizing antibody responses to inactivated VSV with those to two inactivated human pathogenic viruses: highly cytopathic poliovirus (PV) and poorly cytopathic measles virus (MV). The rigidly structured PV efficiently induced neutralizing $\lg M$ antibodies independent of $\mathrm{T}$ cells. In contrast, neutralizing antibodies to the pleomorphic MV were dependent on helper $T$ cells. To test whether this resulted from the differences in virus structure or the capacity of MV to induce cell fusion and/or immunosuppression, we analyzed antibody responses to chimeric MV expressing VSV glycoprotein instead of MV fusion protein and hemagglutinin ${ }^{3}$. IgM antibodies were independent of $\mathrm{T}$ cells; in addition, we found IgG responses dependent on $\mathrm{T}$-cell help that were enduring and protective against lethal VSV infection. Because chimeric MV viruses look like MV ultrastructurally, we conclude that not only structural differences in the envelope but also the ability of MV to induce immunosuppression may limit its capacity to directly activate $B$ cells. These findings are relevant for our understanding of B-cell activation by two prototypic human pathogenic viruses and for the design of new recombinant vaccines.
\end{abstract}

B-cell activation usually requires two signals ${ }^{4}$ : an antigen-specific first signal delivered through the B-cell receptor and a second signal normally provided by cognate T-cell help. There are two antigen classes that activate B cells without need for T-cell help: 'T-independent type 1 ' (TI-1) antigens such as lipopolysaccharide that activate $\mathrm{B}$ cells polyclonally, and ' $\mathrm{T}$-independent type 2 ' (TI-2) antigens, such as repetitive polysaccharides that depend on non cognate signal 2. Many microbes induce TI antibodies: gram negative bacteria by means of lipopolysaccharide (TI-1), and others by means of polysaccharides (TI-2) such as the Streptococcus mutans-derived carbohydrate M1 (ref. 5) or Candida albicans-derived mannan ${ }^{6}$. There is a group of microbes that induce TI antibodies without belonging to either of these categories, which comprises many viruses ${ }^{7-10}$. Vesicular stomatitis virus (VSV), for example, behaves as a TI-1 antigen ${ }^{2}$, although it has no polyclonal activity ${ }^{11}$. Detailed studies revealed that the repetitive and rigid organization of the glycoprotein (VSV-G) in the viral envelope is responsible for efficient cross-linking of B-cell receptors to induce TI- 1 reponses ${ }^{12}$. This may also apply to several other viruses that have a rigid and regular structure ${ }^{13}$.
Here, we analyze neutralizing antibodies to poliovirus (PV) and measles virus (MV) in comparison with VSV (a close relative to rabies virus; Table). PV, a member of the Picornaviridae, has a rigid icosahedral structure. In contrast to VSV, in which the whole envelope consists of about 1,200 identical VSV-G molecules in a paracrystalline array ${ }^{14}$, the PV surface contains four regularly ordered proteins (VP1-4). The crystal structure of PV showed three major clusters where neutralizing antibodies can bind; as in VSV-G, these determinants are 7 to $12 \mathrm{~nm}$ $\operatorname{apart}^{15}$. In contrast, MV, a member of the Paramyxoviridae, forms pleomorphic particles that are very variable in shape and size, with two polymeric proteins, $\mathrm{F}$ (fusion protein) and $\mathrm{H}$ (hemagglutinin), in a fluid layer of the apparently floppy envelope. Neutralizing determinants were found on both proteins, as shown by experiments using recombinant vaccinia viruses that express only one of the proteins and confer protection to mice challenged with MV (ref. 16).

To assess the dependence or independence on T-cell help of IgM and IgG responses, we first immunized BALB/c mice intravenously with UV-inactivated VSV (Fig. 1a), polio vaccine (Salk) (Fig. $1 b$ ) or UV-inactivated MV (Fig. 1c). After 12 to 18 days mice were given booster immunizations with the same dose of virus, and neutralizing antibodies were measured. The early neutralizing IgM response against VSV and PV peaked on day 4, whereas for the response against MV, the peak was reached later, on day 8. Only UV-inactivated VSV was able to induce considerable IgG on primary immunization; PV and MV induced prompt IgG on booster injection. Depletion of $\mathrm{CD}^{+} \mathrm{T}$-cells in vivo (Fig. $1 a-c$, filled symbols) showed that the early IgM response to the rigidly structured PV was TI-type and comparable to that of VSV, whereas the IgM response to MV was obviously dependent on the help of $\mathrm{CD}^{+} \mathrm{T}$-cells. As expected, the IgG responses to all viruses were dependent on $\mathrm{T}$ cells, confirming the well established role of $\mathrm{T}$ cells in immunoglobulin class switching; this result also demonstrated the efficiency of depletion of T cells in vivo in these experiments.

Although MV is not as rigidly structured as VSV and PV, it has polymeric antigens in its envelope that might be able to induce TI antibodies. The following four points may explain the unexpected finding that IgM responses to MV are dependent on $\mathrm{T}$ cells: The independence of $T$ cells of neutralizing antibodies to VSV results from intrinsic B-cell stimulatory properties of VSV-G; on binding to specific B cells, MV induces immediate fusion with the cell at neutral $\mathrm{pH}$ leading to insufficient intensity and duration of B-cell receptor cross-linking to allow TI B-cell activation; the lack of independence of $\mathrm{T}$ cells is related to the pleomorphic 
$a$

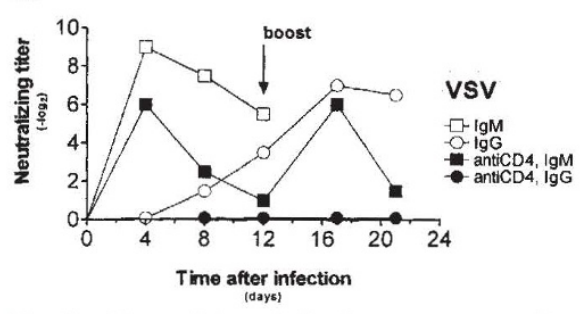

Fig. 1 Neutralizing antibody responses to three different viruses. BALB/C mice were immunized on day 0 with: $\boldsymbol{a}$, inactivated vesicular stomatitis virus (VSV); $\boldsymbol{b}$, poliovirus (PV); or $\boldsymbol{c}$, inactivated measles virus (MV) and 'boosted' after 12-18 days with an equal dose of virus. Serum samples were taken at the indicated time points and analyzed for neutralizing antibodies $b$

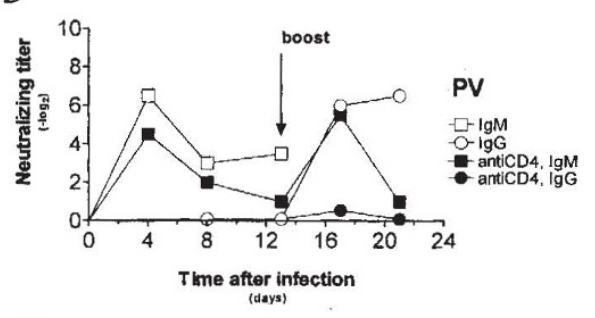

C

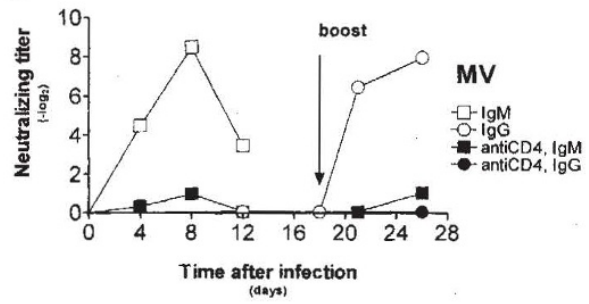

in an in vitro plaque reduction assay. Squares, IgM titers; circles, IgG titers. Groups of mice competent for $\mathrm{CD} 4^{+} \mathrm{T}$ cells (open symbols) were compared with mice depleted in vivo for $\mathrm{CD}^{+} \mathrm{T}$ cells (filled symbols). Each data point represents the mean of three mice per group. Standard deviation was within \pm 1 dilution step. Experiments were repeated once or twice.

floppy envelope structure of MV; and MV envelope proteins induce direct immunosuppression of B cells.

The first explanation seems unlikely because, in contrast to an earlier study ${ }^{17}$, we were not able to detect polyclonal B-cell stimulation either by VSV particles or by recombinant VSV glycoprotein $^{11}$. The second possibility was addressed by immunizing $\mathrm{BALB} / \mathrm{c}$ mice intravenously with inactivated MV either untreated or preincubated in vitro with a monoclonal anti-F antibody. To detect F- or H-specific antibodies in the sera of immunized mice, we did a FACS analysis on L cells (a mouse fibroblast line) expressing MV $\mathrm{H}$ or F proteins. All the neutralizing activity (IgG) in the sera of mice immunized with inactivated MV was specific for $\mathrm{H}$ (Fig. $2 a$ and $b$ ). If the F protein on MV was blocked in vitro by a monoclonal antibody, early IgM (Fig. 2c) as well as secondary IgG (Fig. 2d) antibodies remained dependent on T cells, as shown by comparison of antibody responses between mice deficient in $\mathrm{T}$ cells (nude) or competent for T cells. Therefore, immediate fusion induced by MV, which was already known to be inefficient with mouse cells and which

Table Characteristics of the RNA viruses used

VSV

PV

Rhabdoviridae
Vesiculovirus
2 (IND, NJ)

\section{Picornaviridae Enterovirus \\ 3 (I, II, III)}

- Serotypes

Host

- natural host

- infectibility of

experimental mice

\section{Structure}

- shape

- diameter

- envelope

- surface proteins

Receptors

- main receptor

- coreceptor

- ligand on virus

- cell entry via

Immune response

- neutral. antibodies to

- spacing of neutral. det.

- T cell-dep. of early IgM

- T cell-dep. of lgG

- protection against reinf.

$$
\begin{gathered}
\text { cows } \\
++ \text { (neurotropic) }
\end{gathered}
$$

humans + (adapted virus) humans + (adapted virus)

artificial constructs not tested

\section{Paramyxoviridae Morbillivirus \\ (chimeric viruses) mainly Morbillivirus} none

ponses dependent on $\mathrm{T}$ cells. ineric MVs expressing VSV-G instead of MV F and $\mathrm{H}$ in their en(he exogecytoplasmic tail of F. Both are replication-competent and able infect a variety of rodent and primate cells including Vero cells. ralizing IgM response on day 4, and an efficient switch to IgG after one booster injection. Rough estimation of the amount of VSV-G on the chimeric virus and on VSV by electron microscopy and biochemical analysis ${ }^{3}$ showed comparable antigen quantities in both envelopes. Immunization of BALB/c mice depleted for $\mathrm{CD}^{+} \mathrm{T}$ cells (Fig $3 c$ ) showed that the early IgM anti-VSV response induced by chimeric viruses had become TI (Fig. 3c), whereas the switch to IgG remained dependent on T cells (again confirming the efficiency of the $\mathrm{CD}^{+}{ }^{+} \mathrm{T}$-cell depletion procedure). Expression of only one protein in chimeric virus envelopes, as compared with two different proteins in the MV envelope, seems to enhance antigen repetitiveness and therefore B-cell immunogenicity. However, electron microscopy of the chimeric viruses showed that both chimeric viruses looked pleomorphic, like Edmonston $M V$, despite the fact that they have only VSV-G in their en- 

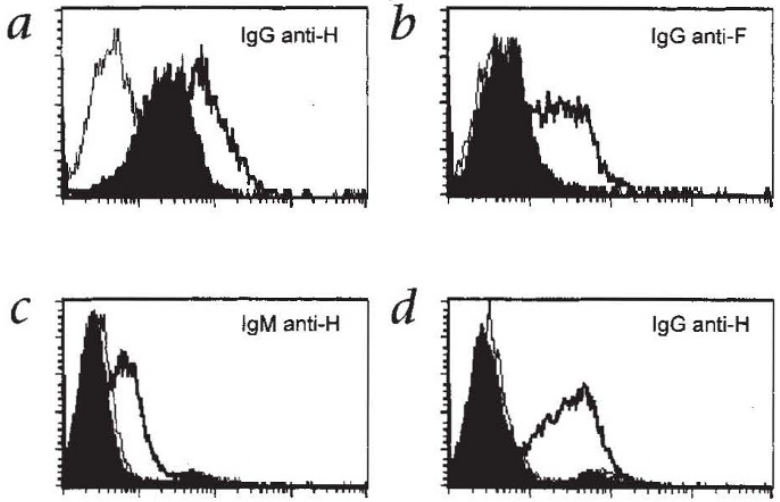

Relative fluorescence intensity $\left(\log _{10}\right)$

velope $^{3}$. These findings, with our in vivo results that replacement of $\mathrm{F}$ and $\mathrm{H}$ by VSV-G restored the TI antibody response, indicate that not only the more floppy envelope structure with two different proteins (instead of only one in the case of VSV or chimeric viruses), but also (and more importantly) direct immunosuppression induced by $\mathrm{H}$ and/or $\mathrm{F}$ (the fourth point mentioned above) may limit the capacity of MV to activate B cells independently of helper T cells. MV induces direct suppression of B cells and immunoglobulin secretion ${ }^{18,19}$. Our results indicate that this effect on $B$ cells is mediated by the MV envelope glycoproteins as has been demonstrated for $\mathrm{T}$ cells ${ }^{20}$, and is probably not caused by the nucleoprotein of MV (ref. 21). Therefore, whether the third or fourth hypothesis is more likely cannot be answered definitively. However, the pleomorphic form of chimeric MV particles and the fact that PV expresses four different proteins in its envelope and still induces TI antibodies because of a highly stable structure make the fourth hypothesis more probable.

Chimeric measles viruses induced enduring neutralizing IgG antibody responses after only two intravenous immunizations with inactivated particles. In a long-term experiment, a stable neutralizing titer could be shown for as long as six months (Fig

Fig. 3 Immune response to chimeric VSV - measles viruses. $\boldsymbol{a}$, Induction of VSV-neutralizing antibodies by UV-inactivated chimeric viruses after primary (day 0 ) and secondary immunization (day 13) with equal doses. Squares, IgM; circles, IgG antibodies. Filled symbols, immunization with MG/FV; open symbols, immunization with MGV. Each data

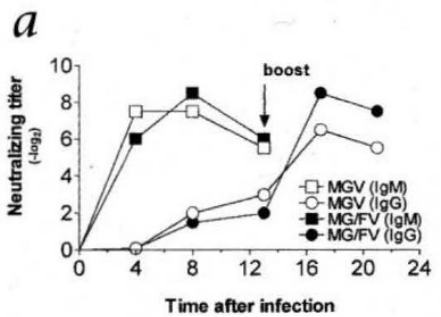
point represents the mean of three mice per group. Standard deviation was within \pm 1 dilution step. The experiment was repeated twice. $\boldsymbol{b}$, Specificity of hyperimmune sera to VSV, MV, MGV and MG/FV in an in vitro plaque or focus reduction assay with the respective viruses. Sera specific for chimeric viruses are able to neutralize the immunizing chimeric virus and VSV, but not MV. c, Role of T-cell help in antibody responses against chimeric viruses. IgM antibodies four days (d4) after primary immunization are independent of $\mathrm{T}$ cells as demonstrated by immunization of mice depleted for $\mathrm{CD}^{+} \mathrm{T}$ cells (open bars), whereas Ig $\mathrm{G}$ antibodies eight days (d8) after booster immunization are dependent on T cells. $\boldsymbol{d}$, Chimeric viruses induce long-lived VSV-neutralizing antibody responses with stable IgC titers up to six months. Each data point represents the mean of three mice per group. Standard deviation was within \pm 1 dilution step. The experiment was repeated once. $\boldsymbol{e}$, Mice deficient for the INF $\alpha$ receptor were immu-
Fig. 2 Specificity and dependence on T cells of MV-specific antibodies analyzed by FACS. $\boldsymbol{a}$ and $\boldsymbol{b}, \mathrm{BALB} / \mathrm{c}$ mice were immunized once with UVinactivated MV. Serum samples were taken on day 19 after immunization and analyzed for $\mathrm{MV} \mathrm{H}$ - or F-specific antibodies by FACS analysis on $\mathrm{L}$ cells transfected with either $\mathrm{H}(a)$ or $\mathrm{F}(b)$. Bold line: positive control (monoclonal anti-H or anti-F antibody, respectively); filled area: serum of immunized mice; thin line: negative control (serum of naive mice). $c$ and $\boldsymbol{d}, \mathrm{BALB} / \mathrm{c}$ mice were immunized once with immune complexes of UV-inactivated $\mathrm{MV}$ preincubated in vitro with a monoclonal anti- $\mathrm{F}$ antibody. Serum was taken on day 9 for $\operatorname{lgM}(c)$ and on day 16 for $\lg G(d)$ analysis. Bold line: immunized normal BALB/C mice; filled area: immunized $B A L B / C$ nude mice; thin line: naive $B A L B / C$ mice.

$3 d)$. To demonstrate the protective capacity of these antibodies, we immunized mice deficient for the IFN $\alpha$ receptor (A129 mice), which are very susceptible to VSV infection ${ }^{22}$, with either of the chimeric viruses, to induce IgG antibodies. These mice were challenged with 1,000 plaque-forming units (PFU) of live VSV (that is, more than $100 \times \mathrm{LD}_{50}$ ). All mice primed with the chimeric viruses survived, wheras all unprimed controls died within six days (Fig. 3e).

Our results indicate that recombinant measles virus technology may be used to generate new vaccines that induce efficient early IgM independent of $\mathrm{T}$ cells as well as enduring and protective IgG antibodies dependent on $\mathrm{T}$ cells, even when the viruses are used as inactivated particles. To our knowledge, these chimeric viruses are the first examples of recombinant viruses that show enhanced immunogenicity compared with the parental measles virus.

\section{Methods}

Mice. Female BALB/C mice and A129 mice deficient for the IFN $\alpha$ receptor were obtained from the breeding colony of the institut für Labortierkunde, Faculty of Veterinary Medicine, University Zürich-Irchel, Zürich, Switzerland. Mice were bred in specific pathogen-free (SPF) conditions and used at the age of eight to twelve weeks. The short-term experiments were done in conventional animal facilities.

Viruses. VSV serotype Indiana (Mudd-Summers isolate) was originally obtained from D. Kolakofsky (University of Geneva, Switzerland) and grown on $b$

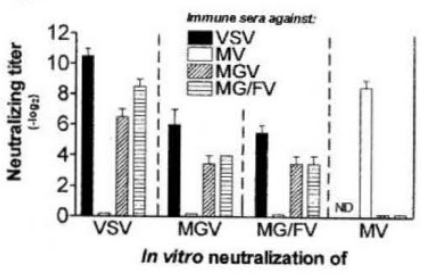

$d$

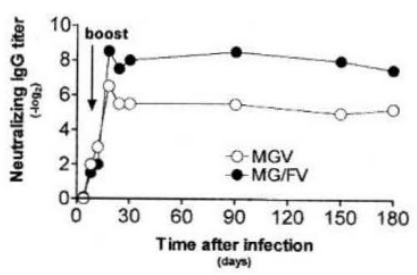

C

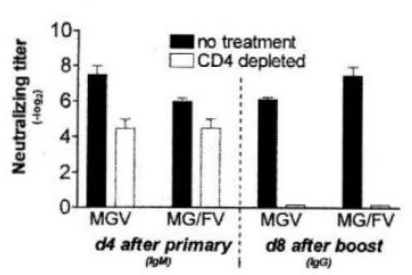

$e$

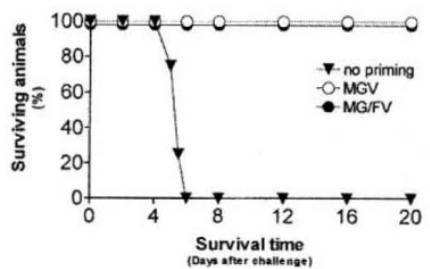

nized twice (with an interval of 12 days) with inactivated MGV or MG/FV or left untreated (no priming). Six days after booster vaccination mice were challenged with live VSV, and survival was monitored for 20 days after the challenge. Four to six mice were used per group. 
BHK cells to virus stocks containing $10^{9} \mathrm{PFU} / \mathrm{ml}$. Poliovirus stock solutions of serotypes I, II and III were obtained from the Swiss Serum and Vaccine Institute (Bern, Switzerland). Poliovirus vaccine was purchased from BERNA (Bern, Switzerland). Measles virus (Edmonston strain) was grown on Vero cells to stocks of $2 \times 10^{6} \mathrm{PFU} / \mathrm{ml}$. Chimeric measles viruses were generated according to protocols described ${ }^{3,23}$ and grown on Vero cells to virus stocks of $3 \times 10^{5}$ to $1 \times 10^{6} \mathrm{PFU} / \mathrm{ml}$.

Inactivation procedures. For UV inactivation, a small volume of high-titer virus preparation was exposed as a thin layer in a petri dish to a UV lamp (15W, Philips) for $3 \mathrm{~min}$ at a distance of $8 \mathrm{~cm}$ (ref. 24). Formalin inactivation of all viruses was done by adding $16 \mu \mathrm{l}$ of $4 \%$ formalin to $1 \mathrm{ml}$ of virus solution (final formalin concentration, $0.0625 \%$ ) and incubation at $4{ }^{\circ} \mathrm{C}$ for 16 $\mathrm{h}$ (ref. 25). Before injection, inactivated virus was diluted with a balanced salt solution, so that $200 \mu \mathrm{l}$ could be injected intravenously. Inactivation was confirmed by plaque assay on Vero cells.

Immunizations, bleeding and in vivo depletion of $\mathrm{CD}^{+} \mathrm{T}$ cells. Mice were immunized intravenously with a standard dose of $5 \times 10^{5}$ to $1 \times 10^{6}$ PFU of inactivated virus on day 0 . For PV experiments, $500 \mu \mathrm{l}$ of polio vaccine (Salk; Poliomyelitis-Impfstoff Berna, Swiss Serum and Vaccine Institute, Bern, Switzerland) was injected into each mouse. Blood for neutralization was taken on days 4,8 and 12 . At the time points indicated (usually after 12 to 14 days), mice were given a intravenous booster immunization with an amount of virus equal to the primary immunization, and blood was taken on days 4 and 8 after the secondary immunization. Before the primary immunization, some of the mice were depleted of $\mathrm{CD} 4^{+} \mathrm{T}$ cells by intraperitoneal injection of two doses of $1 \mathrm{mg}$ of anti-CD4 antibody YTS 191.6 (the hybridoma was a gift from H. Waldmann, Oxford, UK) three and one day before primary immunization, and a third dose on the day before booster injection. Depletion efficiency was checked by FACS analysis of peripheral blood, and $\mathrm{CD}^{+}{ }^{+} \mathrm{T}$ cells were below the detection level.

For preabsorption experiments with $\mathrm{MV}, 10^{7} \mathrm{PFU}$ of UV-inactivated MV was incubated in vitro with $500 \mu \mathrm{g}$ of anti-F antibody for 1 hour at room temperature. Virus-antibody complexes were precipitated with polyethylene glycol 40000 to remove free antibody, and resolubilized in PBS. A dose corresponding to approximately $5 \times 10^{6}$ to $1 \times 10^{7} \mathrm{PFU}$ of MV before precipitation was injected intravenously in each mouse. IgM was determined on day 9 and $\mathrm{IgG}$, on day 16 after primary immunization.

Neutralization assays. Neutralizing titers of sera were determined as described for VSV (ref. 11). The sera were prediluted 20 -fold in MEM supplemented $5 \%$ with $\mathrm{FCS}$, then heat-inactivated for $30 \mathrm{~min}$ at $56^{\circ} \mathrm{C}$. Serial twofold dilutions were mixed with equal volumes of virus diluted to contain $500 \mathrm{PFU} / \mathrm{ml}$. The mixture was incubated for $90 \mathrm{~min}$ at $37^{\circ} \mathrm{C}$ in an atmosphere with $5 \% \mathrm{CO}_{2}$. Serum-virus mixture $(100 \mu \mathrm{l})$ was transferred onto confluent (for VSV, PV and MV) or subconfluent (for chimeric MV) Vero cell monolayers in 96 -well plates and incubated for $1 \mathrm{~h}$ at $37^{\circ} \mathrm{C}$. An overlay of $100 \mu \mathrm{l}$ DMEM containing $1 \%$ methylcellulose was added. After incubation for $24 \mathrm{~h}$ (for VSV), $48 \mathrm{~h}$ (for PV and MV) or $144 \mathrm{~h}$ (for chimeric $\mathrm{MV}$ ) at $37^{\circ} \mathrm{C}$, the overlay was 'flicked off' and the monolayer was fixed and stained with $0.5 \%$ crystal violet. For chimeric MV, plaques were checked directly with a microscope. The highest dilution of serum that reduced the amounts of plaques (for VSV, PV and chimeric MV) or cell fusion foci (for MV) by $50 \%$ was considered the neutralizing titer. Titers are indicated as $-\log _{2}$ of 20 -fold prediluted sera.

FACS analysis. MV immune sera were analyzed by flow cytometry on $\mathrm{L}$ cells expressing either the $\mathrm{H}$ or $\mathrm{F}$ protein of $\mathrm{MV}$. These cells were a gift from F. Wild (Institut Pasteur Lyon, France). They were grown in IMDM supplemented with $10 \%$ FCS and harvested with PBS containing $0.025 \mathrm{M}$ EDTA. Stainings and washings were done in PBS containing $2 \%$ fetal calf serum, $0.2 \% \mathrm{NaN}_{3}$ and $0.025 \mathrm{M}$ EDTA. First cells were incubated with sera prediluted 100 -fold or with monoclonal anti- $\mathrm{H}$ or anti- $\mathrm{F}$ ascites fluid diluted to contain about $10 \mu \mathrm{g} / \mathrm{ml}$ antibody. For detection of specific antibodies, FITC-labelled goat anti-mouse IgG (Sigma) or goat anti-mouse
IgM $^{\mathrm{a}}$ (PharMingen, San Diego, California) antibodies were used. Analysis was done in a FACScan (Becton Dickinson, San Jose, California) using CellQuest software.

\section{Acknowledgments}

We thank $F$. Wild for providing $H$ - and F-transfected $L$ cells and $M V$-specific monoclonal antibodies, and A. Althage for technical assistance. This work was supported by the Swiss National Science Foundation (Grants No 31-32195.91 to R.M.Z. and 31-43475.95 to M.A.B.) and the Kanton Zürich.

\section{RECEIVED 13 JANUARY; ACCEPTED 17 JUNE 1998}

1. Dintzis, R.Z., Middleton, M.H. \& Dintzis, H.M. Studies on the immunogenicity and tolerogenicity of T-independent antigens. J. Immunol. 131, 2196-2203 (1983).

2. Bachmann, M.F., Hengartner, H. \& Zinkernagel, R.M. T helper cell-independent neutralizing $B$ cell response against vesicular stomatitis virus: role of antigen patterns in B cell induction? Eur. J Immunol. 25, 3445-3451 (1995).

3. Spielhofer, P. et al. Chimeric measles viruses with a foreign envelope. J. Virol. 72, 2150-2159 (1998)

4. Bretscher, P. \& Cohn, M. A theory of self-nonself discrimination. Science 169, 1042-1049 (1970).

5. Kimura, S. et al. Streptococcal serotype carbohydrate represents a novel class of type 2 antigen which is T-independent. J. Immunol. 138, 4387-4394 (1987).

6. Mangeney, M., Fischer, A., Le, D.F., Latge, J.P. \& Durandy, A. Direct activation of human B lymphocytes by Candida albicans-derived mannan antigen. Cell. Immunol. 122, 329-337 (1989).

7. Burns, W., Billups, L.C. \& Notkins, A.L. Thymus dependence of viral antigens. Nature 256, 654-656 (1975).

8. Borca, M.V., Fernandez, F.M., Sadir, A.M., Braun, M. \& Schudel, A.A. Immune response to foot-and-mouth disease virus in a murine experimental model: effective thymus-independent primary and secondary reaction. Immunology 59, 261-267 (1986).

9. Charan, S. \& Zinkernagel, R.M. Antibody mediated suppression of secondary IgM response in nude mice against vesicular stomatitis virus. \%. Immunol. 136, 3057-3061 (1986)

10. Szomolanyi-Tsuda, E. \& Welsh, R.M. T cell-independent antibody-mediated clearance of polyoma virus in $T$ cell-deficient mice. J. Exp. Med. 183, 403-411 (1996).

11. Fehr, T. et al. T-independent activation of B cells by vesicular stomatitis virus: no evidence for the need of a second signal. Cell Immunol. 168, 184-192 (1996).

12. Bachmann, M.F., Hengartner, H. \& Zinkernagel, R.M. Neutralizing anti-viral B cell responses. Annu. Rev, Immunol, 15, 235-270 (1997).

13. Bachmann, M.F. \& Zinkernagel, R.M. The influence of virus structure on antibody responses and virus serotype formation. Immunol. Today 12, 553-558 (1996).

14. Thomas, D. et al. Mass and molecular composition of vesicular stomatitis virus: a scanning transmission electron microscopy analysis. J. Virol. 54, 598-607 (1985).

15. Hogle, J.M., Chow, M. \& Filman, D.J. Three-dimensional structure of poliovirus at 2.9 A resolution. Science 229, 1358-1365 (1985).

16. Drillien, R. et al. Protection of mice from fatal measles encephalitis by vaccination with vaccinia virus recombinants encoding either the hemagglutinin or the fusion protein. Proc. Natl. Acad. Sci. USA 85, 1252-1256 (1988).

17. Goodman-Snitkoff, G., Mannino, R.J. \& McSharry, J.J. The glycoprotein isolated from vesicular stomatitis virus is mitogenic for mouse B lymphocytes. J. Exp. Med. 153, 1489-1502 (1981).

18. McChesney, M.B., Fujinami, R.S., Lampert, P.W. \& Oldstone, M.B. Viruses disrupt functions of human lymphocytes. II. Measles virus suppresses antibody production by acting on B lymphocytes. J. Exp. Med. 163, 1331-1336 (1986).

19. McChesney, M.B., Kehrl, !.H., Valsamakis, A., Fauci, A.S. \& Oldstone, M.B. Measles virus infection of B lymphocytes permits cellular activation but blocks progression through the cell cycle. /. Virol. 61, 3441-3447 (1987).

20. Schlender, J. et al. Interaction of measles virus glycoproteins with the surface of uninfected peripheral blood lymphocytes induces immunosuppression in vitro. Proc. Natl. Acad. Sci. USA 93, 13194-13199 (1996).

21. Ravanel, K. et al. Measles virus nucleocapsid protein binds to FcgammaRII and inhibits human B cell antibody production. J. Exp. Med. 186, 269-278 (1997).

22. Müller, U. et al. Functional role of type I and type II interferons in antiviral defense. Science 264, 1918-1921 (1994).

23. Radecke, F. et al. Rescue of measles viruses from cloned DNA. EMBO f. 14, 5773-5784 (1995).

24. Bachmann, M.F., Bast, C., Hengartner, H. \& Zinkernagel, R.M. Immunogenicity of a viral model vaccine after different inactivation procedures. Med. Microbiol. Immunol. (Berl.) 183, 95-104 (1994).

25. Bachmann, M.F., Kündig, T.M., Kalberer, C.P., Hengartner, H. \& Zinkernagel, R.M. Formalin inactivation of vesicular stomatitis virus impairs T- cell- but not Thelp-independent B-cell responses. J. Virol. 67, 3917-3922 (1993). 